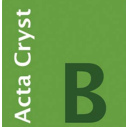

STRUCTURAL SCIENCE CRYSTAL ENGINEERING MATERIALS

ISSN 2052-5206

Keywords: book review; mineralogy; earth and planetary sciences.

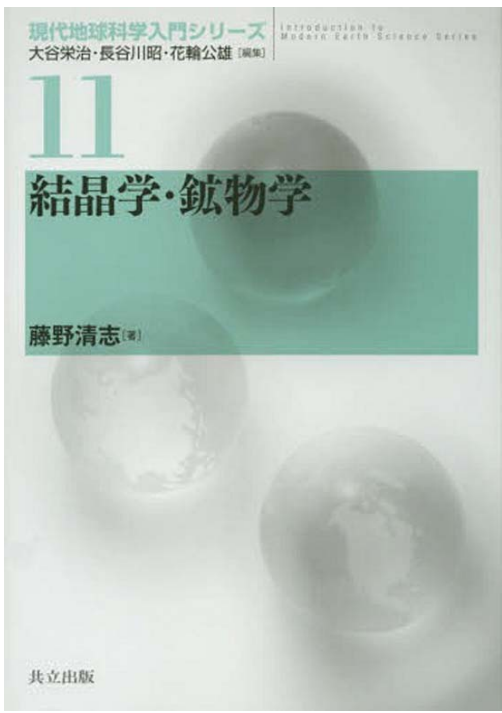

C 2016 International Union of Crystallography

\section{Crystallography and Mineralogy. By Kiyoshi Fujino. Kyōitsu, 2015. Hardcover, X+180. Price Yen 3600. ISBN 978-4-320-04719-8.}

\author{
Massimo Nespolo* \\ Université de Lorraine, CRM2, UMR 7036, Vandoeuvre-les-Nancy, F-54506, France, and CNRS, CRM2, UMR 7036, \\ Vandoeuvre-les-Nancy, F-54506, France. *Correspondence e-mail: massimo.nespolo@crm2.uhp-nancy.fr
}

Crystallography and Mineralogy (kesshōgaku kōbutsugaku), 11th volume in the series Introduction to Modern Earth Science published by the Japanese company Kykōitsu, is a small textbook composed of 11 chapters, three appendices, two appendix tables and one plate, that clearly targets students and young researchers in Earth and Planetary Sciences with a practical approach to the investigation of mineral samples. Given the small size of the book, the topics are necessarily presented in a rather concise way and selected from what could have been a wider choice. The text is well written with a pedagogical approach: a beginner reading this book will have a rather clear idea of the general features of the samples and of what information (s)he may get from the main experimental techniques: for a more in-depth approach one will however need to move to more specialistic textbooks.

The first two chapters, which span only seven pages, summarize the type of samples that are the focus of the treatment and the techniques used for their chemical and structural investigation. Geometric crystallography is the object of chapter 3 ('geometry and symmetry of a crystal', 18 pages), which is the weakest in this book. It makes constant reference to the 1972 edition of International Tables for X-ray Crystallography; for a text appearing in 2015, the choice of such an outdated reference is hard to understand and certainly not justified. A number of imprecisions affect this chapter which, because of the very limited space, reduces to little more than a list of definitions. Note in Table 3.1 we read about a 'trigonal lattice' (sampō kōshi), which simply does not exist, trigonal being a word reserved for a crystal system: the 'other' lattice in the hexagonal family is rhombohedral (hishigata koshi). On the same page, we read that the symmetry of the lattice leads to a classification in a crystal system (shkokei), which is untrue, the result being lattice systems (kōshikei). The same mistakes are repeated on page 18, whereas the figure on p. 19 correctly shows a rhombohedral cell: the contradiction should be selfevident. On p. 12 the definition of geometric element is incorrectly applied to the symmetry element. In the next page a curious listing of eight 'independent symmetry elements' is given, which does not make much sense. Firstly, if the list really concerns symmetry elements, as suggested by the fact that $\overline{3}$ and $\overline{6}$ are missing (being considered the combination of 3 and $\overline{1}$ and of 3 and $m$ perpendicular to it, respectively), then the identity should not appear because it obviously has no 'element'. Secondly, if the list includes symmetry operations instead, as suggested by the presence of the identity, then one could list only 6 and $\overline{1}$, the repeated application of either or both of them generating all the ten types of operations in crystallography point groups. Whatever interpretation is adopted, the statement remains incorrect. Fig. 3.5 in the following two pages gives the stereographic projection of the 32 types of crystallographic point groups where, however, the cubic centrosymmetric groups are given as $m 3$ and $m 3 m$ instead of $m \overline{3}$ and $m \overline{3} m$ (same problem in Table 1 of the appendix): an unjustifiable remnant of prehistoric times when typographical issues primed over consistency ( $\overline{3}$ includes 3 as even powers, whereas the opposite is not true). Table 3.3 in the next page gives [100] instead of [110] as the tertiary symmetry direction of tetragonal crystals; furthermore, only one direction (square brackets) is given instead of a representative set of equivalent directions (angular brackets) for cubic and uniaxial crystals, and the direction chosen does not follow international standards: this certainly comes from the questionable decision of choosing as a reference an old mineralogy book by Morimoto et al. (1975) instead of International Tables. No time to breathe, another imprecision follows immediately: the orthographic 
projection in Fig. 3.6 is called stereographic. After a very short introduction to Bravais types of lattices (where only the Ccentred cell is mentioned, $\mathrm{A}$ and $\mathrm{B}$ being absent), the notion of space group closes the chapter. We remark that the $e$-glide is missing in the list of symmetry elements, but the corresponding graphical symbol occurs twice in Table 3.5. Evidently, with only five pages introducing space groups, the presentation remains cryptic for a beginner and unsatisfactory for the expert. For example, the statement that the general position is away from symmetry elements is partly incorrect (orbits on a screw axis or a glide plane have their multiplicity unaffected and do correspond to the general position); the statement that the multiplicity of the general position is identical to the order of the space groups is misleading (the multiplicity appearing in the space group diagrams is a finite number giving the number of equivalent atoms in the unit cell, whereas the space group has infinite order); the site-symmetry group is described as 'the symmetry elements of the equivalent positions' (the reader will not be able to grasp whether these relate the equivalent atoms or fix them).

The following chapter (24 pages) begins emphasizing the complementarity between X-ray diffraction and electron microscopy in the structural investigation of minerals. Bragg's law shows up without derivation, whereas Laue equations are derived, although briefly. Here a serious overlook may become extremely misleading for the beginner. The statement (page 30) that every atom in a unit cell produces the same lattice and obeys the same diffraction conditions is obviously untrue: otherwise extraordinary crystallographic orbits would not exist and special reflection conditions would never occur (think of $P c c 2$ for example, where an atom on any of the special positions defines a crystallographic orbit with double periodicity along $\mathbf{c}$ and does not contribute to diffractions with odd values of $l$ ). It follows a geometrical introduction of the reciprocal lattice and of the Ewald sphere, where the reader will certainly appreciate the parallel between the X-ray and electron diffraction. Systematic absences and multiple diffractions are the object of the following section (completed by Appendix B), where reflection conditions are incorrectly called 'extinction rule' (in English in the text). These are derived by making the structure factor equal to zero (which is, however, introduced only in a following section), as in the majority of textbooks, ignoring Burger's more pedagogical geometric presentation (Nespolo, 2015). The confusion between a lattice node (which can never 'disappear') and the associated intensity (which can systematically go to zero) witness a lack of terminological rigorousness which is certainly regrettable (the same mistake occurs again in Appendix B, p. 145). Much more convincing is the explication of the annihilation of the systematic absences due to multiple diffraction, where the diffracted beam incident on a second family of planes is seen as coming from a new origin, with respect to which this family no longer corresponds to the systematic absences due to the diffraction of the direct beam. In the next section the determination of point and space group and lattice parameters from diffraction data are discussed in a very short 'how-to-do' manner which does not give enough information to the reader. The curious statement according to which the information about the point group (of the crystal) can be obtained from the 'symmetry of the reciprocal lattice points' leaves the reader puzzled, because a point always has the symmetry of a sphere; what is meant here is the symmetry of the weighted reciprocal lattice (i.e. the intensities associated with the reciprocal lattice; Bricogne, 2010). The following statement about the absolute validity of Friedel's law is a clear anachronism (Flack, 2014). The following section is perhaps a world record of synthesis in that the structure factor, the phase problem and Fourier maps are all squeezed into just three pages: the whole process is described in a rather clear way, but one is naturally led to wonder how useful such an exceedingly concise introduction can be for a true beginner. This is followed, quite naturally, by a discussion of the microstructure that can be observed with a transmission electron microscope, containing a robust description of point, line and planar defects, which is unfortunately affected by the usual confusion between lattice and structure. For example, an illustration of the generation of a phase boundary in Fig. 4.20 would be correct if the black and white circles were atoms; treated as lattice nodes they make part $(c)$ of the figure, where the boundary is shown, hardly meaningful.

The following chapter (nine pages) on spectroscopic analysis presents IR and Raman very briefly (one may ask why reference is made to molecules in a book targeting mineralogists); Mössbauer is presented in much more depth, and a few words about X-ray emission; NMR and ESR are only mentioned but not presented at all.

From chapter 6 we move to mineralogy. This chapter (24 pages) aims at being an extremely concise compendium of mineral systematics, but the space devoted to the topic is insufficient. After a brief introduction on sphere packings, perturbed by the usual nasty confusion between lattice and structure, there is a rapid survey of oxides, sulfides and (a few) silicates. Somewhat surprising is the imbalance between the detailed description of perovskites and post-perovskites (minerals of the mantle) and the extremely limited space devoted to classical rock-forming minerals, where amphiboles, phyllosilicates and cyclosilicates (just to name a few) are mentioned in a list but immediately forgotten. The structural description could have been based on more general principles (like, for example, in Zoltai \& Stout, 1984); here the emphasis being placed mainly on the coordination numbers might mislead the reader into believing that pyroxenes and garnets, both having eight-, six- and four-coordinated polyhedra, have closely related structure, which is of course untrue.

Chapter 7 (13 pages) brings the title 'mineral crystal chemistry', but actually deals with the structure of the atom, the elementary theory of atomic orbitals, the type of chemical bond, ionic radii and coordination number (the latter already used in the previous chapters) to suddenly do a (quantum?) jump to 'simulations' (molecular dynamics, first principles... something is missing in between).

Chapter 8 (11 pages) discusses the stability of minerals in terms of thermodynamics. After a concise enunciation of the three principles and the definition of the fundamental quan- 
tities, the concept of solid solution is introduced with some intuitive guidelines about isomorphic substitutions (Goldschmidt's rules could have been quoted here), followed by the fundamentals of phase diagram construction in terms of minimal free energy. This is followed quite naturally by chapter 9 (16 pages) on phase transformations in minerals, i.e. about morphotropic transformations (according to Groth's definition) which include phase transitions as a special case. The accent is put on the kinetic (diffusional versus diffusionless), mechanistic (reconstructive versus displacive) and thermodynamic (first order versus second order) classification criteria of phase transformations. The limited space does not allow for any extensive treatment, but the presentation is clear, pedagogical and covers fundamental topics such as nucleation and growth, stability and metastability, Avrami's equation, immediately applied to the example of the olivinewadsleyite-ringwoodite transformation, whose importance in the study of the earth's mantle does not need to be emphasized. The chapter ends with the example of a spin transition of iron under the pressure: the treatment is presented in terms of the crystal field theory, which is however not introduced separately almost certainly due to space limitations: one may nevertheless wonder whether a newcomer in the field would take full advantage of such a concise presentation.

Chapter 10 (11 pages) discusses the physical properties of minerals: the stress-strain relation (where one cannot understand why a tensor is called 'constant', both in Japanese and in English, p. 129), electrical and magnetic properties (essentially definitions and brief classification), followed by 2.5 pages on equation-of-state, containing almost as many equations as text (one would implore for more space here!)

The last chapter (eight pages) discusses methods and equipment for synthesizing minerals in the laboratory: hightemperature synthesis, flux method and high-pressure synthesis. Although concise, as is everything in this text, this part is absent in the vast majority of introductory texts and the decision to present something different is certainly welcome.

Appendix A attempts to explain how Hermann-Mauguin symbols change with a change of basis vectors in less than two pages: there is nothing beyond simple examples (we note once again the incorrect use of the symmetry element instead of operation). Appendix B (four pages) lists integral, zonal and serial reflection conditions and shows graphically the effect of multiple diffraction masking the reflection conditions. Appendix C (five pages) discusses the geometry of electron diffraction, with emphasis on the camera constant. Appendix
Table 1 (11 pages) gives a list of space-group types and appendix Table 2 (four pages) lists Shannon and Prewitt's ionic radii. The bibliography (70 titles) and an index close the volume.

Proof-reading is always a hard task: a few typos have remained in the book. In equation 4.12, the 'infinite' symbol occurs instead of 'proportional to'. In the first sentence of section 4.3.3 the radical of the verb 'speak about' is missing ('beta' instead of 'nobeta'). The diopside formula on page 75 contains three oxygen atoms instead of six. A curious typo ('modyfied', in English in the text) occurs on page 117, as well as KIMA instead of KJMA on page 114.

Overall, the main merit of this small book is to make accessible a wide spectrum of notions and concepts to a potentially large public for whom the language barrier can sometimes be a serious problem. Given the small size, it is clear that the author has only touched the surface on many topics. The crystallographic part is affected by many imprecisions and a serious lack of rigorousness (the reference to the 1972 edition of International Tables rather than the most recent one is simply unacceptable); one cannot regret enough the fact that Sadanaga's classical textbook (Sadanaga, 1986) is out of print and rarely available even in the second-hand market. In the mineralogical part a reasonable compromise has been chosen, to present some modern results without repeating a long and sometimes tedious detailed classification which is available in so many textbooks; however, the presentation remains too incomplete.

In conclusion, if a second edition is planned, one can only strongly encourage the author and the publisher to amend all the problematic points raised above, and possibly expand the text somehow to give more breadth to the topics that in this edition suffer from some degree of claustrophobia: the result would then certainly be a text much more useful for a larger audience of beginners in the field.

\section{References}

Bricogne, G. (2010). International Tables for Crystallography, Vol. B, 3rd ed., Ch. 1.3, edited by U. Shmueli. New York: Wiley.

Flack, H. (2014). Chimia, 68, 26-30.

Morimoto, N., Sunagawa, I. \& Miyashiro, A. (1975). Kōbutsugaku. Tokyo: Iwanami shoten.

Nespolo, M. (2015). J. Appl. Cryst. 48, 1290-1298.

Sadanaga, R. (1986). Kesshōgaku josetsu. Tokyo: Iwanami shoten.

Zoltai, T. \& Stout, J. H. (1984). Mineralogy: Concepts and Principles. Minneapolis: Burgess Pub. Co. 